\section{Federico Chopin (1810-1849) y su enfermedad}

\author{
PABLO YOUNG ${ }^{1}$, JORGE M. BERNACIAK ${ }^{1}$, JULIO E. BRUETMAN ${ }^{1}$, \\ BÁRBARA C. FINN ${ }^{1}$, MARCELO C. MIRANDA ${ }^{2}$
}

\section{Frederic Chopin (1810-1849), and his disease}

Frédéric Chopin - a great Polish composer and pianist-suffered from a chronic disease. Both during his life and after his death, physicians disagreed on Chopin's diagnosis. His contemporaries accepted the diagnosis of tuberculosis, a common disease in the 18th century. Description of new clinical entities provoked new dilemmas in the 21th century. Although other alternative diagnoses to tuberculosis have emerged, such as cystic fibrosis or alpha-1 antitrypsin deficiency, we still sustain that the first diagnosis is the most probable. In this paper we report F. Chopin's case history and discuss cons and pros for different diseases as the cause of F. Chopin's suffering and death.

(Rev Med Chile 2014; 142: 529-535)

Key words: Cystic fibrosis; Diagnosis; Genetics; History of Medicine.
'Servicio de Clínica Médica, Hospital Británico de Buenos Aires, Argentina.

${ }^{2}$ Unidad de Neurología, Clínica Las Condes, Santiago de Chile.

Conflictos de intereses: los autores utilizaron el formato propuesto por ICMJE y declararon no tenerlos.

Recibido el 9 de septiembre de 2013, aceptado el 8 de noviembre de 2013

Correspondencia a: Dr. Pablo Young Hospital Británico. Perdriel 74 (1280) Buenos Aires, Argentina. Teléfono: 541143096400 Fax: 541143043393 pabloyoung2003@yahoo.com.ar

\section{Perspectiva histórica}

F ederico Chopin (Figura 1) nació el 22 de febrero de 1810 en Żelazowa Wola, a 60 kilómetros de Varsovia, en Polonia. Su padre, Nicolás Chopin (1771-1844), fue un emigrado francés, y su madre, Tekla Justyna Kryżanowska (1782-1868) perteneció a una familia de la nobleza polaca. Federico fue el segundo hijo y único varón de cuatro hijos ${ }^{1}$.

Federico y sus hermanas crecieron en un ámbito en el que el gusto por la cultura en general, $y$ la música en particular, era considerable. Su hermana Ludwika fue su primera maestra de piano. Al destacar pronto sus excepcionales cualidades, a los seis años sus padres lo pusieron en manos del maestro Wojciech Żywny.

A los siete años de edad, compuso su primera obra: la Polonesa en sol menor para piano, publicada en noviembre de 1817.

A los ocho años tocaba el piano con maestría, y componía: dio su primer concierto público el 24 de febrero de 1818. El resto de su vida musical puede leerse en sus múltiples biografías.

\section{Su obra}

Su legado musical constó de 27 estudios de piano, 25 preludios, 21 nocturnos, 52 mazurcas, 19 polonesas, 4 rondós, 4 scherzos, 4 baladas, 4 impromptus, 2 conciertos, 3 sonatas y una barcarola, entre otros. Su perfecta técnica, refinamiento estilístico y elaboración armónica han sido comparadas con las de Johann Sebastian Bach, Franz Liszt y Ludwig van Beethoven, por su perdurable influencia en la música de tiempos posteriores. La obra de Chopin representa al romanticismo musical en su estado más puro ${ }^{1}$.

\section{Enfermedad crónica de Chopin}

El comienzo de su enfermedad fue reportado por George Sand (1804-1876), su amiga y amante, y también por su amigo Franz Liszt (1811-1886) ${ }^{1-4}$.

En su juventud, Chopin sufrió de procesos respiratorios, diarreas recurrentes y pérdida de peso. En 1826 (a la edad de 16), tuvo una enfermedad que duró 6 meses, durante la cual presentó 


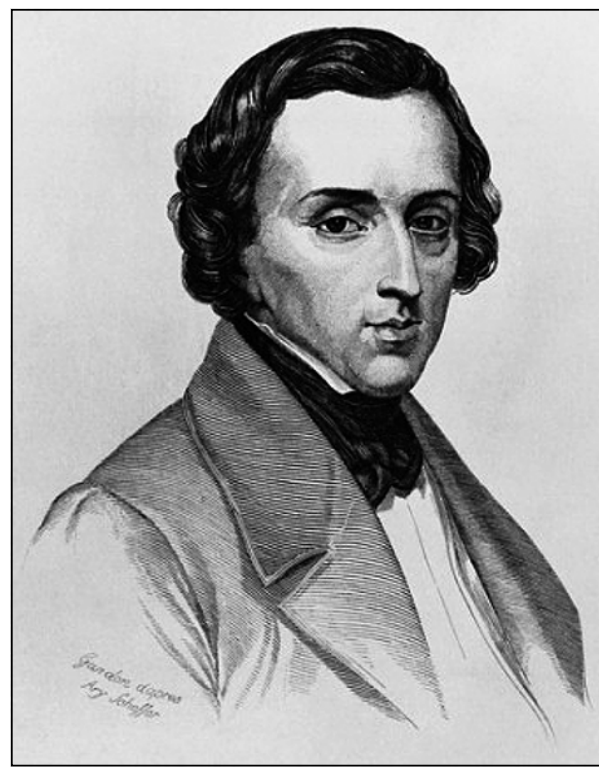

Figura 1. Federico Chopin. En: http://foglobe.com/data_images/main/frederic-chopin/frederic-chopin-03.jpg; [consultado 2/9/2013].

quejas respiratorias, severas cefaleas, y adenopatías cervicales.

Vivió en Polonia y desarrolló su genio musical allí, pero el deseo de ser famoso y aprender más música, asociado con la inestabilidad política en Polonia, lo hizo partir a Francia en $1830^{1}$.

En 1831 (a la edad de 21 años), se quejó de dolor torácico, hemoptisis y cefalea.

Fue notorio que a la edad de 22 años no presentó vello facial, como el mismo describiera en 1832. Nunca fue padre a pesar de los 8 años de relación con George Sand.

En febrero de 1837 enfermó con fiebre alta. Presentó además hematemesis, hemoptisis y permaneció exhausto y apático. Fue confinado a su cama por varias semanas. El Dr. Pierre Gaubert, médico de Chopin, fue inflexible y enfatizó que no sufría de tuberculosis (TBC), y sugirió que un clima templado ayudaría a su estado de salud ${ }^{2}$.

Chopin presentó disnea crónica. Eligió vivir en un clima más suave por lo que se trasladó a la isla de Mallorca y a pesar de ello continuó enfermo. El 3 de diciembre de 1837 escribió, "Estas dos semanas, he estado enfermo como un perro, tuve escalofrios a pesar de una temperatura de $33^{\circ} \mathrm{C}$, entre rosas, naranjos, palmeras e higueras. De los tres doctores más famosos de la isla, uno olió lo que

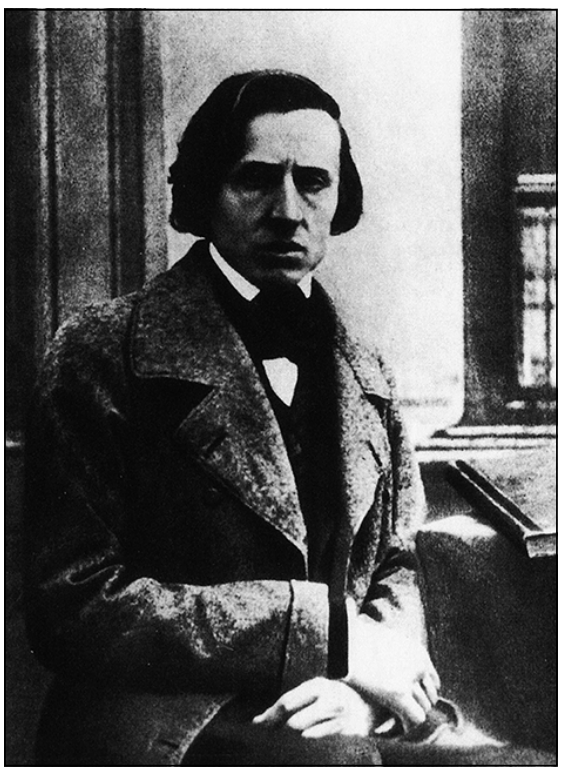

Figura 2. Federico Chopin con palidez apergaminada. En: http://foglobe.com/data images/main/frederic-chopin/ frederic-chopin-02.jpg; [consultado 2/9/2013].

escupí, el otro tapó donde había escupido, el tercero escuchó mientras yo expectoraba. El primero dijo que yo estaba muerto, el segundo que me estaba muriendo y el tercero que yo me moriría"1.

En febrero de 1839 abandonó Mallorca, estableciéndose en Marsella. En septiembre de 1842 regresó a Paris. Los médicos que lo examinaron antes y después de su viaje a Mallorca insistieron en que el compositor no sufría de tuberculosis. Sin embargo, todos los biógrafos de Chopin consideran que la TBC fue la enfermedad que lo afectó desde joven y le ocasionó la muerte ${ }^{2}$.

Su salud declinó visiblemente desde 1840 . Tuvo una pérdida de peso importante y presentó palidez apergaminada (Figura 2). Una caricatura, hecha por su amigo Pauline Viardot en 1844, mostró que Chopin presentó tórax en tonel y piernas extremadamente delgadas (Figura 3).

\section{Los últimos años}

El invierno de 1847 le trajo breves mejorías y prolongadas recaídas, y el término de su relación con George Sand se asoció a la declinación de su salud.

Aunque estaba débil, delgado y pálido, visitó 


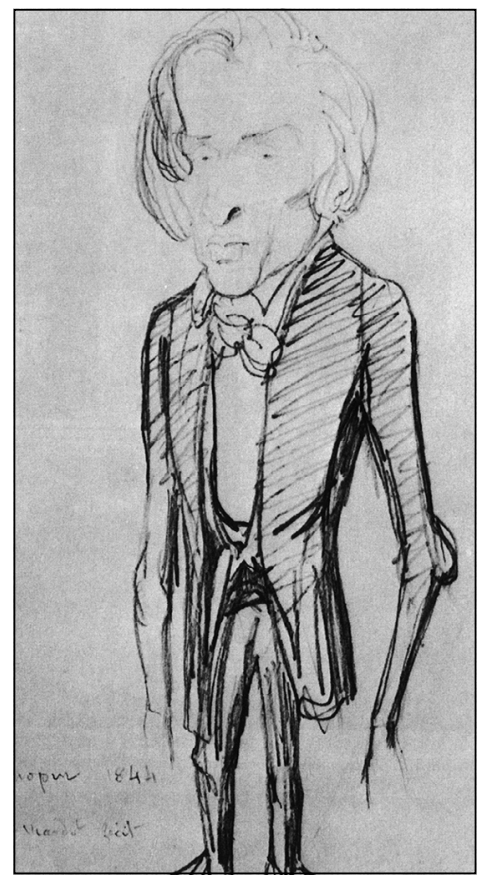

Figura 3. Caricatura de Federico Chopin, hecha por su amigo Pauline Viardot en $1844^{14}$.

Londres. El propósito del viaje era dar conciertos. Ésto combinado con la intranquilidad política en Francia, el apoyo de sus amigos, y el deseo de recuperarse del dolor de su separación de George Sand, fueron suficientes motivos para dejar Paris. En Londres, estuvo confinado frecuentemente en cama, pero en abril se sintió lo suficientemente bien para ir a Escocia, donde estableció una nueva relación, con Jane Stirling. Después de su retorno a Londres (17 de noviembre de 1848), escribió " $\mathrm{He}$ estado enfermo los últimos 18 días, desde que regresé a Londres no he abandonado la casa. He tenido tal resfrío y tales dolores de cabeza, disnea y todos los malos sintomas (mi cabeza me duele mucho aparte de la disnea y la tos). Los doctores me visitan diariamente". Durante el viaje de regreso a Paris escribió a su amiga Solange: "Mañana voy a Paris, apenas me movilizo y más débil que nunca. Estoy hinchado, con neuralgia, no puedo respirar ni dormir" 1,2 .

A su regreso recibió la noticia que su médico predilecto, el Dr. Molin, había fallecido. Desde ese momento su salud se deterioró rápidamente: su estado era tal que apenas podía hablar o levantarse de la cama.

El 22 de junio de 1849, tuvo dos episodios de hematemesis, edema en ambos miembros inferiores, y estaba letárgico. A fines de junio escribió:
"Gracias a Dios no tengo fiebre, lo cual me angustia $y$ enoja a los aprendices de doctores".

El 2 de julio escribió: "No he vuelto a tener hemoptisis desde antes de ayer, mis piernas están hinchadas, estoy débil y haragán, no puedo subir escaleras, me sofoco".

Los años de mayor creatividad fueron justamente aquellos en los que se manifestaron la enfermedad pulmonar y las crisis depresivas pasajeras; esto es, entre los 29 y los 36 años. El propio Federico lo dijo: "En el piano expreso mi desesperación".

\section{Los últimos días}

El período de sus últimos días, aun si confiamos en los testigos presenciales, reúne una mezcla de contradicciones que no es posible desentrañar.

En los primeros días de octubre de 1849, la condición era tal que sin apoyo no podía permanecer sentado y estaba con una gran disnea. Escribió unos pocos días previos a su fallecimiento: "Como esta tierra me sofoca, yo le imploro abrir mi cuerpo, así no seré enterrado vivo".

En la tarde del 15 de octubre de 1849 se sintió muy mal; durante la noche no podía hablar debido a una brusca disfonía y presentaba periódicas pérdidas de conciencia. Al día siguiente dejó instrucciones relacionadas con sus manuscritos. Le pidió a su hermana Ludwika que su corazón fuera enterrado en Polonia y expresó el deseo que en la misa de difuntos se interpretara su Réquiem. Durante la noche tuvo convulsiones (cuya etiología y naturaleza nunca se aclararon) y severos dolores. Entonces durmió hasta la medianoche; cuando despertó le dijo a su médico: "No más". Falleció a las 2 de la mañana del 17 de octubre de 1849. El Dr. Cruveihier constató el fallecimiento. La causa se describió en su certificado de defunción como "Tuberculosis de los pulmones y laringe".

Fue enterrado en el Cementerio de Père-Lachaise, en Paris (Figura 5).

\section{Post Mortem}

La autopsia la realizó el Dr. Cruveilhier, pero su reporte nunca fue hallado. Jane Stirling, en una respuesta a una carta de Liszt manifestó que el Dr. Cruveilhier mencionó: "La autopsia no de- 


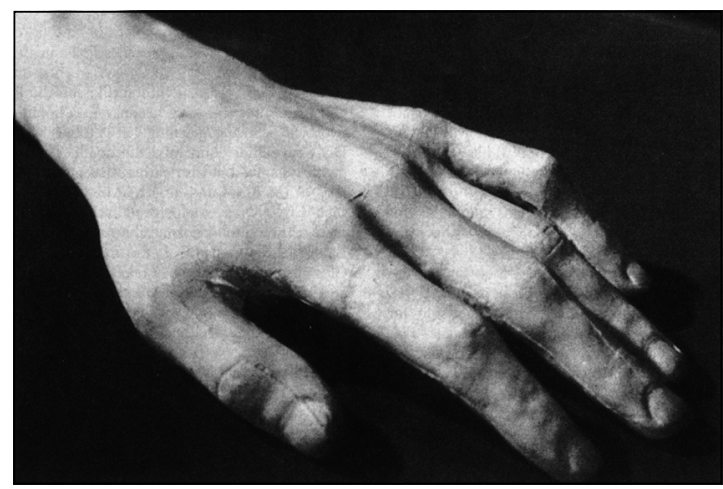

Figura 4. Mano de Federico Chopin. En: http://www.artprints-on-demand.com/a/clesinger-jean-baptiste-a/a-cast-ofthe-hand-of-fre.html; [consultado 2/9/2013].

terminó la causa de su muerte, pero parecía que los pulmones estaban menos afectados que el corazón. Es una enfermedad que nunca había encontrado previamente".

\section{Diagnósticos diferenciales}

Chopin presentó síntomas respiratorios recurrentes (tos, disnea, hemoptisis, cianosis), que comenzaron en su adolescencia; tuvo síntomas sistémicos (poca tolerancia al ejercicio, astenia, cansancio, emaciación, dificultad para ganar peso, palidez, edema periférico, pérdida de masa muscular); refirió síntomas gastrointestinales (diarrea, intolerancia a los alimentos grasos, hematemesis); no presentó dedos hipocráticos (Figura 4); y la característica más prominente de su enfermedad fue su componente respiratorio (adjunto a ello es posible una patología gastrointestinal). Esto podría haber sido causado por las siguientes condiciones:

Déficit de alfa 1 antitripsina: Una posibilidad es que tuviera enfisema secundario a un déficit de alfa 1 antitripsina (ya que no fumaba), que es un déficit congénito metabólico producido por la falta de un inhibidor de la proteasa de la alfa antitripsina ${ }^{5}$. Se asocia con enfermedades degenerativas del pulmón (enfisema panacinar, bronquiectasias, bronquitis crónica e infecciones bronco-pulmonares recurrentes), hepatitis neonatal, cirrosis hepática, falla en el desarrollo e insuficiencia pancreática ${ }^{5-7}$. Podría explicar las recurrentes infecciones pulmonares de Chopin, su pérdida de peso, además de sus síntomas diges-

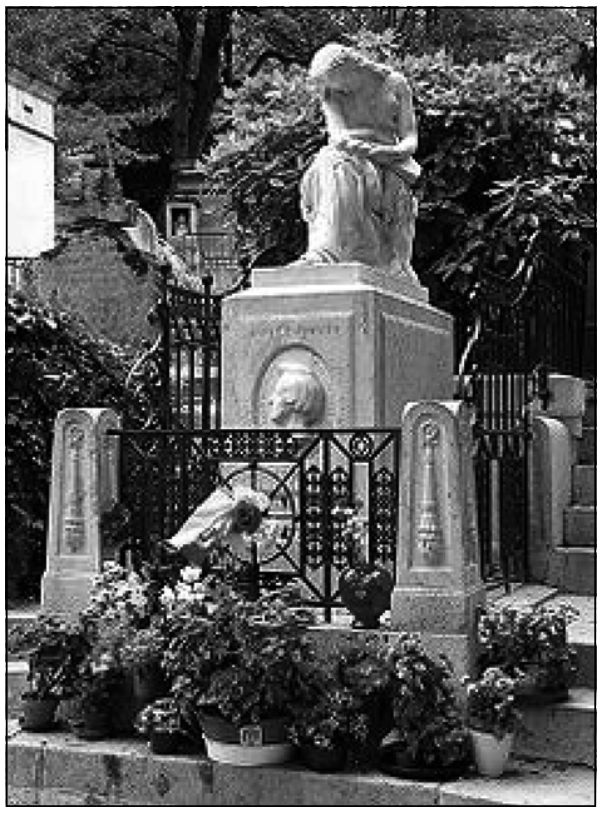

Figura 5. Tumba de Chopin en el Cementerio de Père-Lachaise de París. En: http://es.wikipedia.org/wiki/ Fr\%C3\%A9d\%C3\%A9ric_Chopin; [consultado 2/9/2013].

tivos, todos los cuales eran crónicos y tempranos en su comienzo. Sin embargo, es importante notar que la historia de diarrea crónica asociada con la insuficiencia de alfa 1 antitripsina con insuficiencia pancreática no era obvia.

Kuzemko, al revisar la historia médica, destaca la intolerancia de Chopin a ciertos alimentos, el bajo peso, la falta de vello facial y la infertilidad, y supone la existencia de deficiencia de antitripsina alfa 1, la cual es poco probable porque la gran mayoría de los afectados por esta enfermedad presentan síntomas respiratorios después de los 25 años, mientras que Chopin enfermó del pulmón mucho antes ${ }^{8,9}$. Por otra parte, Cruveilhier nunca habló de enfisema, diagnótico que conocía dado que ya lo había descriptó René-Théophile Hyacinthe Laënnec (1781-1826).

Fibrosis quística (FQ): Esta entidad, como el déficit de alfa 1 antitripsina, produce bronquiectasias, cuya clínica tuvo Chopin. Además de en estos raros cuadros, las bronquiectasias se veían de manera frecuente en la tos convulsa, el sarampión y la TBC. En 1987, John O'Shea concluyó que Chopin no enfermó de TBC y propuso como causa a la FQ o mucoviscidosis, reconocida como entidad clínica hace pocas décadas ${ }^{4}$. La FQ es un desor- 
den multi-sistémico asociado con obstrucción bronquial debido a mucus comprimido y denso. O'Shea mencionó datos de la historia clínica de Chopin, que lo llevaron a afirmar el diagnóstico de $F Q$, como el tórax en barril que es aparente en algunos de los retratos del músico (Figura 3); la delgadez extrema de las piernas; la fragilidad física que le obligaba a tocar el piano suavemente y lo hacía terminar exhausto después de sus interpretaciones; datos sugerentes de insuficiencia pancreática, como su preferencia por una dieta a base de carbohidratos; la supuesta infertilidad del compositor; la intolerancia al calor; la sudoración extrema y, sobre todo, la larga historia de enfermedad pulmonar supurativa. Esta condición se asocia comúnmente con neumonía recurrente. La muerte por sobre infección y cor pulmonale (falla cardiaca derecha) ocurre en la primera década de la vida. Es posible explicar la mayoría de los síntomas y signos de Chopin por una FQ, pero parece poco probable que la padeciera porque en la era pre-antibiótica era muy rara la supervivencia de los pacientes más allá de una edad temprana. Es dable notar, sin embargo, que la FQ es una enfermedad de severidad variable, y por ello es posible que Chopin tuviera una forma más leve de esta enfermedad.

La infertilidad masculina es casi universal en la $\mathrm{FQ}$, pero que Chopin haya tenido una relación de ocho años con George Sand sin hijos no es prueba de esterilidad. Se estima que intimaron aproximadamente por ocho años, entre 1838 y 1846 , pero sólo durante el primer año mantuvieron relaciones. El 12 de mayo de 1846 Sand escribió a un amigo: "Por siete años he vivido como una virgen con él”

En 1996, Steimkamp coincidió con el diagnóstico de FQ. Insistió en varios datos que hacen difícil aceptar el diagnóstico tradicional de TBC: no son compatibles con la TBC la muy prolongada duración de la enfermedad, ni el cuidado de Chopin por ocultar sus manos en público usando siempre guantes, lo que la hizo pensar en la existencia de "dedos en palillo de tambor con uñas en vidrio de reloj" o dedos hipocráticos. Para ella, Chopin padeció una de las formas más benignas de mucoviscidosis ${ }^{11}$. Por otro lado, lo que descarta la posibilidad de FQ es la ausencia de dedos hipocráticos, un hallazgo presente en la casi totalidad de los enfermos de mucoviscidosis que llegan a la edad adulta, que no se aprecia en los moldes de las manos de Chopin (Figura 4$)^{12}$.
Tuberculosis pulmonar: Podría decirse que Emilia (hermana de Chopin) padeciera TBC, aunque no explicaría su muerte por una hemorragia digestiva alta, salvo por una lesión de Dieulafoy o una úlcera péptica. Esta enfermedad pudo haberse transmitido a su hermano.

Es lógico atribuir a la TBC la prematura desaparición de Chopin, ya que a mediados del siglo XIX era la causa más frecuente de enfermedad y muerte en el norte de Europa, al igual que sigue siendo la causa de muerte por infección más importante en el mundo. Sin embargo, de la revisión de las biografías de Chopin surgen varias dudas sobre la aceptación de la TBC como el diagnóstico definitivo. Nuestras inquietudes son las siguientes:

Si padeció TBC durante casi un cuarto de siglo, la duración y el curso de la infección fueron extraordinariamente atípicos. Parece poco probable que sin tratamiento, alguien sobreviviera más de 24 años con una TBC recurrente. A pesar de ello, es posible sufrir esta enfermedad por largos años antes de fallecer. Laënnec, el inventor del estetoscopio tuvo los primeros síntomas de TBC en 1803 y murió en 1826, y como él hay otros ejemplos ${ }^{13}$.

Las hemoptisis recurrentes de Chopin podrían haber sido causadas por $\mathrm{TBC}^{14-18}$. La incidencia de dedos hipocráticos en hombres con TBC pulmonar avanzada es de $40 \%{ }^{19}$. La hemoptisis recurrente es mucho más frecuente en la TBC crónica que en la $\mathrm{FQ}^{12}$.

Sus síntomas gastrointestinales no tienen explicación clara. Es poco probable que tuviera insuficiencia pancreática y viviera mucho tiempo. Quizá la hematemesis fue causada por una enfermedad ulcerosa y la intolerancia a las grasas por una enfermedad calculosa biliar.

El compromiso intestinal es común en la TBC pulmonar crónica y podría explicar parte de sus síntomas. Se sospechó que tuvo cirrosis por la ausencia de vello y la presunta infertilidad, aunque esto último fue refutado. Se ha comunicado la cirrosis cardiotuberculosa de Hutinel-Frield-Pick por pericarditis constrictiva crónica, que se presenta con ascitis, ictericia y edema, las dos primeras no observadas en Chopin ${ }^{20,21}$. Una cirrosis pudo deberse a cor pulmonale crónico, secundario a la hipoxia por su enfermedad pulmonar. Si bien se dijo que tuvo cirrosis, eso no queda claro de la descripción clínica y la ausencia de vello facial no es un indicador.

En el certificado de defunción, Cruveilhier 
escribió: "TBC pulmonar y laríngea". Pero, aparentemente la autopsia arrojó otro diagnóstico (el informe se perdió). Él fue una de las grandes autoridades en la patología de la TBC y comentó que el músico no la había sufrido y que el corazón estaba más dañado que los pulmones ${ }^{22}$. Si este es el caso, su creencia debe ser tenida en cuenta muy seriamente, porque era una autoridad en anatomía patológica y tuberculosis ${ }^{14,17}$. El resto de los médicos que asistieron a Chopin a lo largo de su vida (aproximadamente 30) no lo consideraron tísico y él mismo, durante la mayor parte de su vida, no aceptó ser tuberculoso. Laënnec publicó en 1819 (Chopin tenía 9 años) su libro De L'auscultation médiate, donde describe diferentes sonidos percibidos con el estetoscopio que él mismo inventó ${ }^{23}$. Con esto queremos hacer notar que el examen físico era ya una herramienta poderosa para el diagnóstico, en Francia, y ninguno de los médicos que lo atendió diagnosticó TBC. Posiblemente tuvo una forma no cavitada. Jane Stirling, en respuesta a una carta de Liszt, manifestó que el Dr. Cruveilhier mencionó "la autopsia no determinó la causa de su muerte, pero parecía que los pulmones estaban menos afectados que el corazón. Es una enfermedad que nunca había encontrado previamente" y lo mismo mencionó Ludwika Chopin. Estos escritos los tomamos con pinzas por ser una evidencia indirecta.

Los episodios de disfonía pudieron deberse a una TBC laríngea. Franken y Absolon, luego de una larga evaluación, manifestaron como diagnóstico una TBC sistémica, o sea laríngea, intestinal y pulmonar ${ }^{1}$.

Recordando que es más probable que un paciente tenga una enfermedad común con presentación atípica, en vez de una enfermedad poco común, y que si el médico elige el diagnóstico menos probable se equivoca la mayoría de las veces, creemos que el diagnóstico más probable de la enfermedad de Chopin es una TBC de larga data y serían menos probables una forma incompleta de FQ o un déficit de alfa 1 antitripsina ${ }^{24}$.

Chopin fue un genio que cambió la forma, la utilización de la técnica y el estilo del piano y también revolucionó las formas musicales. Un genio que superó, hasta límites sobrehumanos, la adversidad de padecer una enfermedad física debilitante y un temperamento inestable.

Es importante recordar que el diagnóstico retrospectivo es complejo, ya que emitimos una opinión sin haber visto al paciente ${ }^{25}$. En los tiempos que corren, el diagnóstico es indispensable para establecer el tratamiento y el pronóstico.

El diagnóstico patográfico (descripción de enfermedad) retrospectivo corre el riesgo de restringir la historia al proceso biológico no teniendo en cuenta el contexto social ${ }^{25}$.

Después de 164 años no es posible conocer la naturaleza de la enfermedad de Chopin, pero las conjeturas sobre los distintos problemas de salud son un ejercicio intelectual fascinante. Tal vez, en un futuro próximo, el análisis genético de los restos de Chopin y de sus familiares más cercanos puedan proporcionamos la certidumbre que hoy nos elude.

Agradecimientos: Al Dr. Alberto Agrest, por la lectura crítica y aportes a este manuscrito.

\section{Referencias}

1. Franken FH, Absolon KB. Diseases of famous composers. Rockville: Editorial Kabel Publishers; 1996. p. 173-87.

2. Neumayr A. Frederic Chopin. En: Neumayr A. Music and medicine: Chopin, Smetana, Tchaikovsky, Mahler. Notes on their lives, works and medical histories. Bloomington, Illinois, EEUU: Editorial Press Media; 1997. p. 11-137.

3. O'Shea JG. Music and Medicine. London: Editorial JM Dent; 1993. p. 140-55.

4. O'Shea JG. Was Frédéric Chopin's illness actually cystic fibrosis? Med J Aust 1987; 147: 586-9.

5. Stoller JK, Abboussouan LS. Alpha-1 antitrypsin deficiency (Review). Lancet 2005; 365: 2225-36.

6. Shin MS, Ho JK. Brochiectasis in patients with alpha 1-antytrypsin deficiency: a rare ocurrence? Chest 1993; 104: 1384-86.

7. Al Kassimi F. Bronchiectasis and homozygous alpha1antitrypsin deficiency. Thorax 1996; 51: 228.

8. Kuzemko JA. Chopin illnesses. J Roy Soc Med 1994; 87: 769-55.

9. Snider G. Pulmonary disease in alpha-1-antitrypsin deficiency. Ann Intern Med 1989; 3: 957-8.

10. Howe MJ. George Sand: The search for love. New York: Editorial The John Day Company; 1927. p. 269.

11. Steinkamp G. [Did Frédéric Chopin actually suffer from tuberculsis?]. Pneumologie 1996; 3: 807-11.

12. di Sant'Agnese PA, Davis PB. Cystic fibrosis in adults: 75 cases and a review of 232 cases in the literature. Am J Med 1979; 66: 121-31.

13. Nuland SB. Doctors: The biography of medicine. New York: Vintage Books; 1989. p. 200-37. 
14. Kubba AK, Young M. The long Suffering of Frederic Chopin. Chest 1998; 113: 210-6.

15. Cheng TO. Chopin's illness. Chest 1998; 114: 654-5.

16. Carter ER. Chopin's malady. Chest 1998: 114: 655-6.

17. Margolis ML. The long suffering of Frederic Chopin, revisited. Chest 1998; 114: 655.

18. Cheng TO. Chopin's illness revisited. Chest 1998; 114: 1796.

19. Reeve PA, Harries AD, Nkhoma WA, Nyangulu DS, Wirima JJ. Clubbing in African patients with pulmonary tuberculosis. Thorax 1987; 42: 986-7.

20. Osler W. The principles and practice of medicine. 3rd ed. New York: Editorial D Appleton and Co; 1899. p. 319-21.
21. Sharma MP, Bhatia V. Abdominal tuberculosis. Indian J Med Res 2004; 120: 305-15.

22. Park RHR, Park MP. Caput Medusae in medicine and art. BMJ 1988; 297: 1677-9.

23. Roguin A. Rene Theophile Hyacinthe Laënnec (17811826): the man behind the stethoscope. Clin Med Res 2006; 4: 230-5.

24. Eddy DM, Clanton $\mathrm{CH}$. The art of diagnosis, solving the clinicopathological exercise. N Engl J Med 1982; 306: 1263-8.

25. Karenberg A. Retrospective Diagnosis: Use and Abuse in Medical Historiography. Prague Medical Report 2009; 110: $140-5$. 